\title{
Passive transfer studies in demyelinating neuropathy with IgM monoclonal antibodies to myelin-associated glycoprotein
}

\begin{abstract}
ANDREAS J STECK, ${ }^{*}$ NORMAN MURRAY,${ }^{*}$ JEAN-CLAUDE JUSTAFRE, ${ }^{*}$ CLAUS MEIER, $\dagger$ KLAUS V TOYKA $\ddagger$ KURT HEININGER, $\ddagger$ GUIDO STOLL
\end{abstract}

From the Departments of Neurology, Universities of Lausanne* and Bern, $\uparrow$ Switzerland and the Department of Neurology, University of Düsseldorf, $\ddagger$ Federal Republic of Germany

SUMMARY Serum or IgM fraction from two patients with a demyelinating neuropathy and IgM monoclonal antibodies to myelin-associated glycoprotein were injected in three different animal species. There were no clinical, electrophysiological or morphological signs of demyelination in either chronic or acute passive transfer experiments. These results suggest that the pathogenesis of this human demyelinating neuropathy may be more complex than has been assumed.

Several recent reports have described a demyelinating neuropathy in the setting of an IgM gammopathy with antibody activity to a myelin antigen referred to as myelin-associated glycoprotein. ${ }^{-3}$ This well established clinical association, together with the presence of wide separation of the myelin lamellae that has been shown systematically in these cases, ${ }^{24}$ suggests that the demyelination may reflect antibody mediated damage to the peripheral nerve. To test this assumption we have performed passive transfer experiments. We wish to report our inability to induce a neuropathy in both chronic and acute passive transfer experiments.

\section{Patients and methods}

Serum was obtained from two patients ( $\mathrm{PB}$ and $\mathrm{AK}$ ) with a demyelinating neuropathy associated with a monoclonal IgM gammopathy and from one control with IgM gammopathy without neuropathy. Clinical and pathological data have been described elsewhere. ${ }^{25}$ The monoclonal immunoglobulins from the two patients with neuropathy had proven specificity for myelin-associated glycoprotein. ${ }^{26}$ For chronic experiments, the human IgM was prepared from the sera by euglobulin precipitation followed by gel filtration on sepharose $4 \mathrm{~B}$ (Pharmacia) in $0.1 \mathrm{M}$ Tris- $\mathrm{HCl} \mathrm{pH} 8 \cdot 0,0 \cdot 15 \mathrm{M} \mathrm{NaCl}$. Before injection the material was sterilised by membrane filtration.

Address for reprint requests: Dr Steck, Department of Neurology, Centre Hospitalier Universitaire Vaudois, 1011 Lausanne, Switzerland.

Received 28 December 1984. Accepted 9 February 1985
Chronic experiments: (1) Two adult guinea pigs received an initial dose of human $\operatorname{IgM}(40 \mathrm{mg} / \mathrm{kg})$, the test animal received IgM from patient $P B$ while the other animal served as control. One day later the animals received an immunosuppressive dose of cyclophosphamide (300 mg/ $\mathrm{kg})$. Thereafter the animals received intraperitoneal injections of human IgM $(40 \mathrm{mg} / \mathrm{kg})$ every third day during 20 weeks. Blood samples were obtained by cardiac puncture after 6 and 20 weeks of treatment to determine the levels of circulating human IgM by rate immunonephelometry (Beckman) and to test for anti-myelin-associated glycoprotein activity. ${ }^{2}$ Motor nerve conduction velocity was measured at both sciatic nerves with the anaesthetised animals.' Measurements were made every 2 weeks.

(2) One adult marmoset (Callithrix jacchus) received doses of human IgM (patient PB), ranging from $300-800 \mathrm{mg} / \mathrm{kg}$, every other day during 13 weeks. The injections were given into the dorsal musculature and in part subcutaneously. At the end of the experiment blood was taken to determine the level of circulating human IgM. Motor nerve conduction velocity was measured as described ${ }^{7}$ every week.

(3) In an effort to improve the accessibility of the target antigen to the injected $\operatorname{IgM}$, newborn rabbits were used. Four, 2-day-old rabbits housed with the mother received intraperitoneal injections of human IgM $(50 \mathrm{mg} / \mathrm{kg})$ every other day for 4 weeks. The two test animals received IgM from patient AK while the other two served as controls. At the end of the experiment blood was taken from the animals to determine the levels of circulating human IgM and to test for anti-myelin-associated glycoprotein activity.

At the end of all of the above experiments animals were killed and the sciatic nerves were rapidly dissected and 
prepared for immunocytochemistry using the PAP method $^{\mathrm{s}}$ and for light and electron microscopy.

Acute experiment: Six adult rabbits were anaesthetised and, after lumbar laminectomy, they received a single intrathecal injection of human serum $(0.3$ to $0.5 \mathrm{ml})$. Two test animals were injected with serum from patient $\mathrm{PB}$, two with serum from patient AK and two with control serum. Animals were killed after 3 or 10 days. The lumbo-sacral spinal roots and the cauda equina were removed and prepared for immunocytochemistry using the PAP method and for light and electron microscopy.

\section{Results}

All of the animals injected with human IgM on a chronic basis were found to have detectable quantities of human IgM in the circulation, levels ranged from $1 \mathrm{~g} / \mathrm{l}$ in the experiment with guinea pigs to
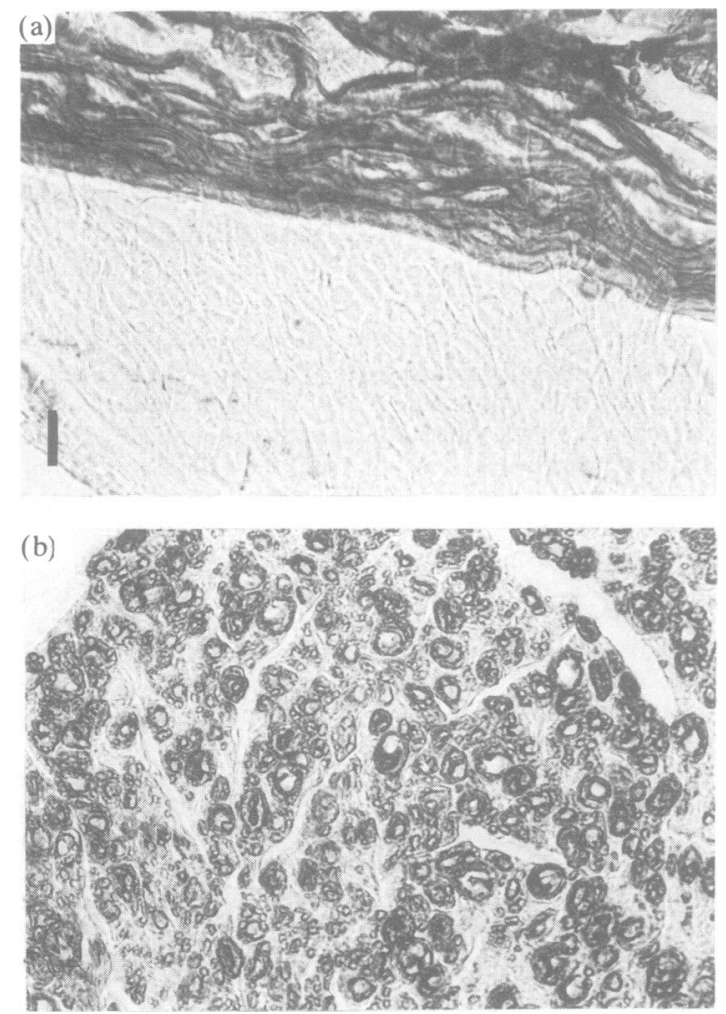

Fig (a) Paraffin section of monkey sciatic nerve after passive transfer of human monoclonal IgM antibody to myelin-associated glycoprotein. Immunocytochemical localisation of the transferred $\operatorname{lgM}$ by the PAP technique. After incubation with rabbit IgG antibody to human IgM (1:500), black reaction product occurs only at the perineurium but not in the myelin. Bar $=20 \mathrm{~nm}$. (b) Section of same sciatic nerve as (a) after post-embedding incubation with human monoclonal antibody to myelin-associated glycoprotein (1:2000). Myelin is intensively stained. $\times 500$.
$15 \mathrm{~g} / \mathrm{l}$, or more, in the experiments with rabbits and monkey. Immunoblotting studies confirmed that theo sera of the test animals contained human antibody气 to myelin-associated glycoprotein while the sera of the control animals did not.

None of the animals involved in acute or chronic@ passive transfer experiments showed neurologicalo. deficit during the observation period. Elec $\overrightarrow{2}$ trophysiological measurements made during the experiments (with guinea pigs and monkey) failed tow show any slowing of motor nerve conduction veloc-O ity. Light and electron microscopy revealed no evi-흘 dence of nerve damage suggestive of $a \bar{s}$ polyneuropathy. Teased fibre preparations dis- $\stackrel{\mathbb{Q}}{\odot}$ played no evidence of paranodal or segmentalos demyelination. Direct immunocytochemical studies? performed on paraffin sections of sciatic nerve? (chronic experiments) or lumbo-sacral roots (acute $\overrightarrow{\vec{H}}$ experiment) failed to reveal deposits of human IgMo in nerve myelin. In the monkey but not the other species, these studies showed the IgM to be locatedo at the perineurium (fig a). In contrast, indirect ${ }_{\infty}^{+}$ immunocytochemical studies confirmed that theio human anti-myelin-associated glycoprotein Igtis bound specifically to nerve myelin in the species used for these experiments (fig b).

\section{Discussion}

Passive transfer of serum from patients with auto immune diseases such as myasthenia gravis" Lambert-Eaton syndrome, ${ }^{10}$ has been used demonstrate the pathogenic role of circulating $\bigodot_{\infty}$ immunoglobulins. Although a pathogenic role of ${ }^{r}$ monoclonal anti-myelin-associated glycoprotein IgM antibodies found in patients with a demyelinating neuropathy has been postulated, this has not yet been positively demonstrated, except for a preliminary report $^{11}$ where intraneural injections were $\mathbb{Q}^{\mathbb{Q}}$ used, which is technically questionable. We chose $\vec{F}$ therefore the passive transfer of intraperitoneally or 3 intramuscularly applied immunoglobulins, an approach which has been shown to be effective in producing morphological and electrophysiological changes in peripheral nerves of animals receiving monoclonal IgM from patients with myelomatous:polyneuropathy. ${ }^{2}$ For these transfer experiments we have used convenient laboratory species in which myelin-associated glycoprotein has been shown to윽 crossreact with the human monoclonal IgM. ${ }^{6}$ Our findings highlight the problem of accessibility of the relevant target antigen, myelin-associated glycopro- $-\frac{7}{O}$ tein, to the large IgM molecules in the circulation. Thus, animals injected with human anti-myelin- $\tilde{N}$ associated glycoprotein IgM were found to have detectable quantities of human IgM in the circula- 
tion and to have significant circulating anti-myelinassociated glycoprotein activity. In spite of this, however, no evidence of IgM deposits in nerve myelin could be demonstrated. This finding is in sharp contrast to the situation in patients with antibodies to myelin-associated glycoprotein in whom IgM deposits on the myelin sheaths have been described. ${ }^{313}$ In this context it is perhaps relevant that patients with non-malignant, as well as with malignant (Waldenström's macroglobulinaemia) gammopathy show changes in endoneurial microvasculature, ${ }^{14}$ and such changes have been observed in patient $\mathrm{AK} .^{5}$ It is conceivable that these changes may increase vascular permeability and improve antibody access to the nerve. In the experiments described here we have attempted to improve the access of the IgM to its target antigen. One approach involved chronic administration of $\mathrm{IgM}$ to newborn rabbits, during a period of one month. In these young animals the blood-nerve barrier may not be fully developed and the anti-myelin-associated glycoprotein IgM will be in the circulation during the period of rapid myelination. Our results show, however, that even under these circumstances the IgM does not penetrate the nerve. Another approach involved intrathecal administration of the serum. With this approach, however, only a single dose was given and again we found no evidence of IgM binding to myelin or to any other structure in the lumbo-sacral roots. The absence of an acute effect of anti-myelin-associated glycoprotein IgM may not be wholly unexpected in view of the very slowly progressive character of the neuropathy seen in the patients.

Recent evidence suggests that the human monoclonal IgM antibodies which recognise myelinassociated glycoprotein react with a carbohydrate epitope and it has been reported that this epitope is shared by a ganglioside of peripheral nerve. ${ }^{15}$ This ganglioside could conceivably be an important target antigen involved in the demyelination but further studies concerning its chemical identity, localisation in nerve and species restriction are required to clarify this. In conclusion the pathogenic potential of human anti-myelin-associated glycoprotein IgM remains to be demonstrated but our findings suggest that the pathogenesis of this human neuropathy is more complex than has been assumed.

This study was supported by grants from the Swiss Multiple Sclerosis Society, the Swiss National Science Foundation, the Deutsche Forschungsgemeinschaft and the Minister for Science and Research, NRW.

\section{References}

' Braun PE, Frail DE, Latov N. Myelin associated glycoprotein is the antigen for a monoclonal IgM in polyneuropathy. J Neurochem 1982;39:1261-5.

${ }^{2}$ Steck AJ, Murray N, Meier C, Page N, Perruisseau G. Demyelinating neuropathy and monoclonal IgM antibody to myelin associated glycoprotein. Neurology (NY) 1983;33:19-23.

${ }^{3}$ Stefansson K, Marton L. Antel JP, et al. Neuropathy accompanying IgM $\lambda$ monoclonal gammopathy. Acta Neuropathol 1983;59:255-61.

${ }^{4}$ Melmed C, Frail DE, Duncan I, et al. Peripheral neuropathy with IgM kappa monoclonal immunoglobulin directed against myelin-associated glycoprotein. Neurology (NY) 1983;33:1397-405.

${ }^{5}$ Meier C, Roberts K, Steck A, Hess C, Miloni E, Tschopp L. Polyneuropathy in Waldenström's macroglobulinaemia: Reduction of endoneurial IgMdeposits after treatment with chlorambucil and plasmapheresis. Acta Neuropathol 1984;64:297-307.

' Steck AJ, Murray N, Vandevelde M, Zurbriggen A. Human monoclonal antibodies to myelin associated glycoprotein. J Neuroimmunol 1983;5:145-56.

${ }^{7}$ Heininger K, Liebert UG, Toyka KV, et al. Chronic inflammatory polyneuropathy. Reduction of nerve conduction velocities in monkeys by systemic passive transfer of immunoglobulin G. J Neurol Sci 1984; 66: 1-14.

* Sternberger LA. Immunocytochemistry (2nd ed). New York: John Wiley, 1979.

" Toyka KV, Drachmann DB, Pestronk A, Kao I. Myasthenia gravis. Passive transfer from man to mouse. Science 1975; 190:397-9.

${ }^{10}$ Lang B, Newsom-Davis J, Wray D, Vincent A, Murray N. Autoimmune aetiology for myasthenic (EatonLambert) syndrome. Lancet 1981;ii:224-6.

"Haus AP, Takatsu M, Latov N, Sherman WH. Focal demyelination of cat sciatic nerve induced by intraneural injection of serum from patients with polyneuropathy and monoclonal IgM reactive with myelin associated glycoprotein. J Neuropathol Exp Neurol 1983;42:349.

12 Besinger UA, Toyka KV, Anzil AP, Neumeier D, Rauscher R, Heininger K. Myeloma neuropathy: Passive transfer from man to mouse. Science 1981;213:1027-30.

${ }^{13}$ Meier C, Vandevelde M, Steck A, Zurbriggen A. Demyelinating polyneuropathy associated with monoclonal IgM paraproteinaemia. J Neurol Sci 1983;63:353-67.

14 Powell HC, Rodriguez M, Hughes AC. Microangiopathy of vasa nervorum in dysglobulinaemic neuropathy. Ann Neurol 1984; 15:386-94.

is Ilyas AA, Quarles RH, MacIntosh TD, Dobersen MT, Trapp BD, Dalakas MC, Brady RO. IgM in a human neuropathy related to paraproteinaemia binds to a carbohydrate determinant in the myelin-associated glycoprotein and to a ganglioside. Proc Natl Acad Sci USA 1984;81:1225-9. 